\title{
Comprendiendo las limitaciones de la investigación
}

\section{Understanding the Limitations of Research}

\author{
Edwin Salas-Blas
}

Universidad San Martín de Porres, Lima, Perú

Universidad San Ignacio de Loyola, Lima, Perú

ORCID: https://orcid.org/0000-0002-0625-0313

\section{*Correspondencia}

Email: e.salasb@hotmail.com

\section{Citar como:}

Salas-Blas, E. (2019). Comprendiendo las limitaciones de la investigación. Propósitos y Representaciones, 7(SPE), e424. doi: http://dx.doi.org/10.20511/pyr2019.v7nSPE.424

(C) Universidad San Ignacio de Loyola, Vicerrectorado de Investigación, 2019. 


\section{Resumen}

Este trabajo es un ensayo argumentativo, en los términos de Montero y León (2005) es un estudio teórico, su objetivo es plantear críticamente una evaluación del estado en el cual se encuentra la investigación psicológica en el Perú, y, a partir de ese análisis plantear los potenciales motivos de la situación problemática y reflexionar sobre dichos aspectos, para plantear finalmente ideas de solución a los problemas detectados. Por sus propósitos y por el discurso que se maneja en el mismo, podría ser útil para estudiantes o equipos de trabajo que deseen realizar investigaciones más profundas, empíricas (cuantitativas o cualitativas) o no empíricas, acerca de la investigación psicológica en el Perú.

Palabras clave: Investigación psicológica en el Perú; Inversión peruana en investigación; Universidad e investigación.

\section{Summary}

This work is an argumentative essay, or, in the terms of Montero and León (2005), it is a theoretical study. Its objective is to critically propose an evaluation of the state in which psychological research is in Peru, and from this analysis, raise the potential reasons for the problematic situation and reflect on these aspects, in order to finally propose ideas for solving the problems detected. For its purpose and the kind of discourse that is managed in it, this could be useful for students or work teams that wish to conduct deeper, empirical (quantitative or qualitative) or non-empirical research, about the topic of psychological research in Peru.

Keywords: Psychological Research in Peru; Peruvian Investmen in Research; University and Research.

\section{Introducción}

El presente trabajo es el resultado de varias exposiciones en eventos académicos en los que el autor participó para tratar el tema de la investigación psicológica en el Perú, especialmente en el II Congreso Internacional de Evaluación Psicológica: Perspectivas y desafíos de la Psicología Educativa, organizado por la Carrera de Psicología de la Universidad San Ignacio de Loyola, realizado en noviembre de 2018. En este evento el autor expuso el tema bajo el título La Ley Universitaria (Ley 30220 - Julio 2014): Análisis y respuestas a las exigencias en torno a la investigación. Este trabajo podría ser clasificado como un ensayo argumentativo o una forma de estudio teórico (Montero \& León, 2005), cuyo objetivo es reflexionar en torno a la situación de la investigación psicológica en el Perú, plantear críticamente las potenciales causas del problema y desarrollar con brevedad algunas ideas de solución. Podría ser útil para quienes quieran desarrollar estudios empíricos o revisiones acerca del tema.

Si se pregunta ¿cuál es el estado de desarrollo de la investigación psicológica en el Perú?, seguramente una mayoría de investigadores responderán con una visión crítica y estará de acuerdo en que a pesar de esfuerzos aislados de algunos psicólogos notables que merecen el reconocimiento por su labor, tal como los mencionan Alarcón (2006); Arias (2011, 2012, 2014); Caycho (2012, 2013a, 2013b); León (1993a, 1993b); Zanolo (2014), entre otros; después de más de 60 años de formar psicólogos, que se inicia a partir de 1955 con la creación de la Sección de Psicología en la Universidad Nacional Mayor de San Marcos [UNMSM] y en 1963 con la creación del Departamento Académico de Psicología en la UNMSM (Alarcón, 1980, 2000, 2012; Jauregui, 2002); la investigación psicológica básica ni aplicada se han desarrollado como se habría esperado. Para comprender este problema se plantea analizar el contexto en el cual la psicología y los psicólogos investigadores se desenvuelven, tomando en cuenta algunos aspectos que podrían servir luego para desarrollar investigaciones más rigurosas y puntuales, se propone el análisis de: el papel del estado en torno a la investigación científica, algunas características de 
una cultura que no investiga, el rol de la universidad y de la Superintendencia Nacional de Educación Superior Universitaria [SUNEDU], la situación de las publicaciones científicas y algunos planteamientos para revertir la situación.

\section{El Estado Peruano y la investigación científica}

¿Cuál es el papel del Estado como entidad que debe apuntalar la investigación científica?, un primer aspecto a revisar es la inversión que éste realiza para alentar el desarrollo científico y tecnológico. El discurso político permite encontrar que en casi todos los planes de gobierno presentados por candidatos a gobernar el país (de los que llegaron y no llegaron al poder) ha existido la idea de apoyar el desarrollo científico y tecnológico con miras a lograr el ansiado desarrollo nacional, pero si se revisan los presupuestos anuales de todos los gobiernos se encontrará que la inversión en investigación no ha sido precisamente la más adecuada para lograr ese propósito declarado antes de llegar al gobierno, peor aún si se verifica lo ejecutado del presupuesto.

Consistente con los datos del Banco Mundial (2017) sobre la inversión en investigación en el mundo, Andina (2019), la agencia peruana de noticias reveló que según un funcionario del Banco Interamericano de Desarrollo (BID), en el 2019, la inversión peruana en investigación alcanzó el $0.12 \%$ del producto bruto interno (PBI). Dato que el Sistema Integrado de Administración Financiera del Estado (SIAF), contradice desde años atrás afirmando que en realidad sólo se gasta poco más de la mitad de lo presupuestado (0.08\% del PBI). Es posible, además, que gran parte de ese gasto se relacione con la administración de las instituciones encargadas y poco con el hecho de alentar y mejorar la investigación científica y el desarrollo tecnológico.

Comparada la inversión peruana con lo que realizan otros países del mundo e incluso sólo los latinoamericanos, se encuentra que Perú invierte muy poco y en la región está en la zaga, Colombia lo hace con un $0.25 \%$, Chile con un $0.38 \%$, México con un $0.54 \%$ y una economía más grande como la de Brasil con un 1.24\%. Si se desea desarrollar y lograr una economía en crecimiento, la inversión en investigación debería ser superior al $0.7 \%$. Esto explica por qué los países vecinos, sin estar en las condiciones más ideales, nos aventajan en producción científica. Rebossio (2013a), periodista e investigador de estos temas para el Diario El País de Argentina, señala que la poca inversión en desarrollo científico correlaciona positivamente con la cantidad de científicos que cada país tiene, con la cantidad de patentes que registra cada país y con las exportaciones que realizan de tecnología, etc. En todos estos indicadores nuestro país está a la zaga del mundo e incluso de Latinoamérica.

Esta falta de inversión, que por cierto no es cosa nueva (Bermúdez, 2013), ocasiona numerosos problemas que no permiten desarrollar la investigación, entre ellos, la fuga de talentos, la falta de instituciones encargadas de hacer investigación, falta de personal capacitado, muy pocas publicaciones científicas en las diferentes áreas del conocimiento humano, etc. La Dra. Orjeda, ex presidenta del CONCYTEC sostenía en 2017, que los gobiernos peruanos tuvieron a la ciencia, a la investigación e innovación tecnológica, en un estado de abandono completo, y, que el Perú necesitaba invertir alrededor de S/. 4,000 millones de soles (en dólares algo así como $\$ 1,250$ millones) para atraer, por lo menos, a 17,000 doctores que se requería en ese momento para establecer una política de desarrollo científico acorde a los tiempos de crecimiento económico (Taipe, 2016).

\section{Cultura e investigación}

Un segundo aspecto que se plantea para el análisis es que culturalmente el Perú, desde sus orígenes coloniales, es una sociedad poco preocupada por el desarrollo de conocimiento científico a partir de la investigación empírica. El modelo de universidad que se instaló en el Perú es el modelo de la clásica universidad española, una institución preocupada por las letras y no por la 
investigación que conduzca al desarrollo científico ni tecnológico, hecho que parece una característica común a la mayor parte de países latinoamericanos y que en el caso peruano no se ha logrado superar hasta ahora mismo en que la SUNEDU observa a gran parte de las universidades por su poca vocación para investigar y por la ausencia de cuadros docentes dedicados a dicha actividad -siempre declarada como uno de sus fines más importantes-. En Perú el desarrollo de conocimientos y la producción académica son escasos, las universidades priorizan la formación de profesionales y se descuida la formación de investigadores. Alarcón (2012) plantea que los psicólogos, en una sociedad tercermundista como la peruana, viven en una situación de alienación y de dependencia cultural, sostiene "Vivimos entre productos ideológicos y materiales, valores e instrumentos, no producidos por nosotros, que muy a menudo no se articulan con nuestra realidad humana, social..." (p. 41).

Ni el Perú ni Latinoamérica han participado de los grandes descubrimientos científicos y de las revoluciones científicas, de la manera en que se tuvo la revolución industrial que nació en Europa, la revolución tecnológica que vino desde Norteamérica, y tampoco ahora se participa de la nueva revolución denominada la sociedad del conocimiento ni de la revolución tecnológica que ahora mismo se vive (Rivera, 2011); esto puede tomarse como sinónimo de atraso y a la vez de dependencia que ha ido creciendo más fuertemente en los últimos decenios y que puede agudizarse aún más.

La investigación no se utiliza como un medio para resolver los problemas del país, sean de cualquier tipo. En educación se operan reformas, se aplican nuevas metodologías y estrategias para trabajar y aprender, pero sin conocer la realidad. Lo mismo sucede en cualquier otro sector de la vida del país, se compran aparatos muy modernos que luego no se utilizan cabalmente, o se construyen edificaciones, carreteras, sistemas de regadío, puentes, etc., sin tener en cuenta las condiciones de la naturaleza que luego las destruye. El Perú es dependiente en términos de conocimientos científicos y de tecnología. Rebosio (2013b) afirma que la inversión en investigación correlaciona inversamente con la cantidad de recursos que se gasta en la compra de tecnología y de maquinarias, la menor inversión en investigación e innovación (PBI) se corresponde con mayores gastos en adquisiciones de maquinarias y de tecnología en general.

\section{Universidad e investigación}

Un tercer punto de análisis es el rol de la universidad y de la investigación para el desarrollo del país. Asunto sensible y que lamentablemente tampoco puede ser positivamente evaluado. La universidad peruana en su conjunto no tiene mucho que mostrar como aportes al desarrollo del conocimiento científico y tecnológico, evidentemente existen hechos aislados de aportes de algunos científicos en diferentes áreas de las ciencias y de la psicología en particular, como lo han señalado Alarcón (2006), Arias (2011, 2012, 2014), Caycho (2012, 2013a, 2013b), Leon (1993a, 1993b) y Zanolo (2014), para referirse a los aportes de Blumenfeld, Alarcón, Gonzáles Moreyra, Pollit, entre los más reconocidos; que pretendieron -desde sus propias perspectivas- construir una psicología científica para entender al hombre peruano. Trabajo que finalmente no prosperó, como sí ha sucedido en Colombia y en México, para citar solo dos casos muy conocidos. Lamentablemente, el trabajo de ese conjunto de psicólogos se vio frustrado por falta de recursos, porque no tuvo el eco necesario en su momento o porque cuando ellos dejaron de producir investigaciones, no tuvieron un equipo que continúe con el esfuerzo de un trabajo sistemático, institucional, de conjunto, de equipo que funciona con un plan de largo plazo. Algunos se refieren a esta situación como un alejamiento y aislamiento de la universidad con respecto a las necesidades de la sociedad (Alarcón, 2000; Salas, 2000), al punto que se podría dar por cierto que en Perú, universidad y sociedad viven de espaldas y hasta puede afirmarse que no tienen una visión común del camino y de las acciones que hay que tomar para lograr el desarrollo del país.

Con la nueva ley universitaria (Ley 30220, Julio 2014) y con la acción de control que viene ejerciendo la $S U N E D U$, la investigación está constituyéndose en un rubro importante de la evaluación que este organismo realiza de las diferentes instituciones universitarias; como 
consecuencia, todas las instituciones universitarias tienen la preocupación por presentar indicadores de investigación y de publicaciones, algunas están formando sus propios institutos de investigación, desarrollan políticas de incentivos para publicaciones de sus docentes, están considerando docentes investigadores que por ley tienen un trato especial y promueven la publicación de artículos en revistas indexadas, otras universidades tienen una política de incentivar a quienes, docentes o no, deciden publicar con la pertenencia institucional.

En el momento existe un incremento de investigadores jóvenes en algunas universidades, las publicaciones de estos también han crecido notablemente, hay más interés por mejorar la calidad de las pocas revistas existentes e indexadas. Pero, pocas universidades tienen líneas claras de investigación, pocos son los investigadores que desarrollan su trabajo investigativo y su producción en torno a un tema y se especializan en él.

Si se tiene en cuenta la cantidad de universidades que forman psicólogos (más de 40) y posiblemente algunos miles de psicólogos que hacen docencia en la universidad, la cantidad de psicólogos certificados por el CONCYTEC es muy baja (cerca de 80 investigadores psicólogos). Esto permite afirmar que posiblemente hay instituciones en las que no existe un psicólogo docente investigador que tenga la experiencia de publicar en revistas indexadas y esté certificado por CONCTEC. Esto trae otro problema muy difícil de manejar, ¿cuántos de los docentes que tienen a su cargo las asignaturas de investigación en diferentes universidades, tienen experiencia de investigación y publicaciones en revistas indexadas?

Un asunto relacionado que no está siendo apreciado en su real dimensión, es la calidad de las investigaciones psicológicas en las instituciones universitarias, la mayor parte de ellas carece de trascendencia y no necesariamente están relacionadas con los temas y problemas que el país necesita (Alarcón, 2012), pero, es necesario reiterar que tampoco el estado y la empresa son capaces de tomar iniciativas para coordinar con la universidad líneas de investigación en las cuales estén interesadas. Estado y empresa, esperan que la universidad produzca ciencia y tecnología, pero sin hacer ninguna inversión y eso, lamentablemente, no es posible.

Finalmente, otro aspecto problemático es que a partir de la nueva ley universitaria se exige la presentación y sustentación de investigaciones que se realizan para obtener grados académicos. Se exige para el optar por el Bachillerato, luego por la licenciatura, seguido de la maestría y finalmente en el doctorado. No existen diferencias claramente señaladas para los trabajos de cada uno de los niveles, de modo tal que cada universidad está proponiendo sus propias reglas y exigencias, muchas de ellas discutibles y que podrían en el futuro cercano generar más problemas en el proceso de graduación y titulación.

\section{La situación de las publicaciones científica}

Actualmente la información científica se da a conocer a través de revistas, ellas están insertadas en bases de datos, catálogos e index que las agrupan y clasifican para hacerlas más visibles para los investigadores, para que sean útiles para el desarrollo científico y también para evaluar el impacto de las publicaciones científicas. Algunas de estas bases de datos como Web of Science (WOS) o Scopus tienen mucho prestigio en el mundo académico y científico, y son de difícil acceso para las publicaciones latinoamericanas que tienen poca producción científica y dentro de esto, el Perú está bastante rezagado (Medina, 2014, 2015), lejos de Brasil, Argentina, Chile, Colombia y México, que, como ya se dijo, son los países que más invierten en investigación en la región

El portal de Scimago Journal Rank (SJR) mide el impacto de las revistas científicas y establece la calidad de sus publicaciones fundándose en el recuento de citas obtenidas (información de Scopus). En el ranking de producción científica por países en el año 2018 (www.scimagojr.com/), el país con más producción científica es EE. UU que produjo 683,003 documentos, seguido por China con 599,386. En el puesto 14 se encuentra a Brasil con 81,742 
documentos, luego México (puesto 28), Argentina (44), Colombia (47), Ecuador (62) y Perú en el puesto 70 con 3,369 documentos. Esto tiene correlación positiva con lo que cada país invierte en investigación y también con lo que vende en tecnología y patentes. Luego no tiene mucho sentido seguir preguntándose por qué EE. UU y China son las más grandes potencias del mundo o por qué Brasil es la primera potencia en América Central y del Sur.

Según el reporte de Scimago del 2018 se cuentan 24,701 revistas en Scopus o WOS (SJR www.scimagojr.com/), de las 100 revistas mejor calificadas, 94 pertenecen a EE. UU o al Reino Unido, cinco son holandesas y una de alemana, a pesar de la gran producción china, todavía sus investigaciones no alcanzan las mejores calificaciones en el Ranking de Scimago. Las revistas latinoamericanas son 832 (3.37\% del total) la mayor parte son brasileñas y sólo se encuentran siete revistas peruanas ( $0.84 \%$ del total latinoamericano), una de psicología que pertenece a la Pontificia Universidad Católica del Perú (PUCP).

SciELO es una biblioteca virtual creada en Latinoamérica, en ella se puede encontrar más revistas peruanas, pero solamente tres revistas de psicología, a saber, la Revista de Psicología, de la Pontificia Universidad Católica del Perú; Liberabit, de la Universidad de San Martín de Porres y Propósitos y Representaciones, de la Universidad San Ignacio de Loyola.

En el Sistema Regional de Información en Línea para Revistas Científicas de América Latina, el Caribe, España y Portugal (LATINDEX), se contabilizan 23 revistas peruanas de psicología. Aproximadamente la mitad pertenecen a escuelas o facultades de psicología, lo cual indica que existen algo así como dos tercios de instituciones universitarias que forman psicólogos y que no tienen una revista científica en la que sus docentes puedan publicar, hecho evidentemente contradictorio con la exigencia que ellas mismas plantean a sus docentes. Estas cifras también son claras demostraciones de lo que se viene sustentando, la crítica situación de la investigación, la productividad científica peruana y las revistas científicas.

El problema de gran parte de las revistas científicas peruanas es la sostenibilidad y la calidad de su producción, muchas revistas son creadas con entusiasmo, publican algunos números y dejan de hacerlo porque no tienen sostenibilidad, algunos gestores de revistas científicas no se preocupan por lograr indexaciones y su manejo no tiene en cuenta las recomendaciones de las bases e índices en las que se tienen que incorporar si se desea entrar y tener presencia en el mundo globalizado; tal vez este sea uno de los principales problemas de las revistas nacionales y de su poca visibilidad; pocas revistas tienen equipos bien constituidos por académicos que entienden bien este mundo de las publicaciones científicas (Romero-Torres, Acosta-Moreno \& TejadaGómez, 2013). Muchas revistas peruanas no se encuentran en bases de datos o índices abiertos ni siquiera en los del propio ámbito latinoamericano (Clase, Latindex, Lilacs, Redalyc, etc.), por lo que resulta casi imposible saber de ellas, este es un problema álgido que debería abordarse desde Concytec -al igual que en Colombia lo hace COLCIENCIAS- en el más breve plazo.

Los hechos que se han tratado caracterizan la realidad de la investigación en el Perú y traen consigo una serie de problemas y carencias que influyen directamente en la falta de producción científica y tecnológica, no solamente en psicología, sino en todas las disciplinas. La condición necesaria para revertir esta situación es que exista una mayor inversión pública y/o privada en investigación, y luego se pueden realizar algunas acciones para revertir esta situación.

1. Es necesario formar más psicólogos investigadores calificados. En abril de 2016 CONCYTEC hizo público una primera relación de 200 investigadores calificados, este hecho, más la presión de la SUNEDU a las universidades para lograr mayor compromiso con la investigación han incrementado notablemente ese listado, pero aún resulta insuficiente para las necesidades que el país requiere satisfacer, se requieren más investigadores y se requiere más inversión pública y privada. 
En este momento, la relación de psicólogos reconocidos como investigadores por CONCYTEC es cercana a 80, este número es insuficiente, incluso si solamente se tuviera que cubrir las plazas docentes en las más de 40 instituciones universitarias que forman psicólogos.

Un aspecto importante resulta el aspecto económico, el pago de los investigadores y la dedicación a la actividad de investigar, tienen que mejorar. Idealmente se debe profesionalizar la investigación, con ingresos más decorosos y con mayor tiempo para desarrollar la investigación. Actualmente los sueldos de los docentes son muy variados y dependen de las instituciones (sobre todo en las universidades privadas), y la cantidad de horas que dedican los investigadores a otras labores es una exigencia muy alta, que distrae o perturba la producción científica.

2. Crear institutos y centros de investigación de fenómenos psicológicos. El Perú es un país pluricultural, con docenas de lenguas, con condiciones climáticas y geográficas diversas y costumbres también diferentes. Todo esto afecta el comportamiento de los peruanos y no se ha hecho nada sistemático para investigar esa realidad. No se conoce la psicología del peruano, sea adulto, adolescente o niño; hombre o mujer; de la costa, de la sierra o de la selva. No se tiene idea clara de las formas cómo aprenden los niños de cada región del país, tampoco de lo que los motiva, o de la forma cómo percibe la realidad, de sus valores y estilos de vida. Esto se explica porque se carece de instituciones estatales dedicadas a la investigación y que tengan sostenibilidad.

Alarcón (2012) sostiene que para salir de la dependencia cultural, teórica, científica e instrumental en el que la psicología peruana se halla es necesario utilizar la investigación de manera activa y a la vez paciente, “...una investigación psicológica ligada a los problemas que requiere el cambio social y el desarrollo de nuestros pueblos" (p.41).

3. Definir las líneas de investigación y orientarlas a entender y resolver los problemas sociales. Pocas instituciones universitarias tienen definidas sus líneas de investigación, estas han surgido más por las preferencias temáticas de los investigadores y docentes; $y$, no se relacionan necesariamente con las características del contexto en el cual operan las instituciones universitarias. La investigación psicológica no responde a las necesidades de lo que se desea conocer para solucionar los problemas que como país se tiene, por ejemplo, no se tiene idea de cómo enseñar o motivar a los niños para que aprendan lenguaje o matemática, a pesar de que las calificaciones en PISA los escolares peruanos ocupan los últimos lugares y esto no ha cambiado desde la primera medición realizada hace 30 años aproximadamente; tampoco se tiene idea clara por qué el poblador peruano tiene tanta tolerancia a la violencia o a la corrupción, por citar sólo dos hechos.

Es conveniente relacionar la investigación psicológica con las necesidades de alcance nacional o regional, los organismos del estado como los ministerios, organismos reguladores y empresas públicas y privadas tienen que crear las condiciones para determinar cuáles son los principales problemas psicológicos a los que se enfrentan e investigar, antes de desarrollar intervenciones o programas preventivos. Esto permitiría, por otro lado, desarrollar líneas de investigación básica y aplicada, con objetivos claros y sostenibles, con equipos de trabajo que apunten a la solución de los problemas; e.g. ¿cómo se mejora el aprendizaje escolar de la matemática y del lenguaje?, o, ¿cómo se forman niños honestos que rechacen la corrupción, el engaño?; o ¿cómo enseñar a los niños valores humanos como la igualdad de derechos, el respeto y la tolerancia?, etc.

4. Fortalecer la publicación de las investigaciones que se desarrollen. Una de las decisiones que se debe adoptar en todas las universidades y centros de investigación, es que las investigaciones que se realizan, por docentes e investigadores o por estudiantes, de pre o de posgrado, sean publicadas siempre en revistas o a texto completo en los repositorios institucionales, eso evitaría que se repita el mismo tema de estudio en muchas instituciones y también que se evite el plagio. 
Es evidentemente importante que se aliente a los investigadores peruanos para publicar en revistas Scopus, este es un anhelo que para el investigador peruano se hace muy trabajoso y difícil; al existir en Perú solo una revista de psicología en esta base de datos, las posibilidades de publicar en revistas de esta base, se limita a buscar posibilidades en el extranjero y en gran parte de ellas con tiempos largos para la revisión y aceptación de los manuscritos.

Por otro lado, se tiene que realizar esfuerzos para mejorar la calidad de las revistas, para profesionalizar a los editores y revisores y aspirar que en el tiempo más breve se tengan más revistas y con mejores indexaciones. Evidentemente, se requiere de un organismo como CONCYTEC que se proponga actuar como un abanderado en este trabajo.

5. Capacitar a los psicólogos para desarrollar investigaciones y para afrontar el reto de publicar. Gran parte de los profesionales que desarrollan actividades profesionales y de docencia, no tienen una buena formación metodológica y no han hecho investigación en su formación académica (Merino-Soto \& Salas-Blas, 2016), de modo tal que tienen problemas en varios momentos del proceso de investigación: en el momento de plantear un proyecto de investigación y precisar el problema de estudio, cuando se busca información y se estructura la teoría, en el diseño metodológico y estadístico, y, lógicamente, cuando se hace el informe y el artículo. Cada institución tiene que desarrollar programas de capacitación para sus docentes, y seguramente de a pocos se irá mejorando la calidad de lo que se produzca. La falta de manejo metodológico y el desconocimiento de cómo se desarrolla la investigación son de acuerdo con Merino y Salas (2016) dos de los principales problemas del docente que no investiga.

\section{Conclusiones}

La falta de cultura científica de la sociedad peruana, que involucra a gestores de gobierno nacional, regional, local y de todo tipo de instituciones públicas y privadas; a la clase política como a los propios académicos en las universidades; determina que en el Perú no se desarrollen investigaciones para conocer sus problemas y a partir de allí proponer soluciones para sus necesidades. Posiblemente este mismo factor cultural, tenga que ver también con la distribución del presupuesto, que descuida la investigación. Esto también trae como consecuencia que no se relacione el desarrollo nacional con la investigación, el país, en los últimos 20 años, ha experimentado un crecimiento económico sostenido, pero la inversión en investigación no ha crecido como debería, a pesar de que existen evidencias en el mundo de países que han hecho saltos al desarrollo económico, científico y tecnológico sobre la base de inversión sostenida en investigación.

La carencia de instituciones que desarrollen investigación de la realidad nacional es evidente, esto imposibilita tomar decisiones adecuadas para solucionar los problemas más apremiantes. Esto en el campo de la psicología es muy notorio, no se tiene líneas de investigación ligadas al desarrollo nacional (p. ej. En educación, en salud, en el campo laboral o en seguridad), sino que se genera más dependencia cuando se investigan objetos de estudio que podrían ser irrelevantes para el medio peruano, con teorías provenientes del extranjero e instrumentos que provienen de otras realidades.

Las universidades tampoco tienen tradición investigadora, la Dra. Orjeda (Taipe, 2016), preguntada si las universidades peruanas realizan investigación, responde que los dedos de una mano le sobrarían si quisiera contarlos. Los pasos que se están dando a través de la acción de la SUNEDU, tienen que hacerse más consistentes en la exigencia de que las universidades tienen que realizar investigación.

Asimismo, no basta alentar a los académicos para realizar investigación, con proponer políticas de incentivos; a eso hay que agregarle la necesidad de desarrollar revistas con una buena visibilidad e indexación, se tiene que generar una política de desarrollo de publicaciones científicas en todas las disciplinas. 
En psicología, se tiene que alentar la capacitación de los profesionales para desarrollar investigación y para aplicar el conocimiento resultante en la solución de los diferentes problemas en los que tiene capacidad y competencia para resolverlos. Alentar la construcción y validación de instrumentos para estudiar los diferentes problemas urgentes como en algún momento lo planteó Gonzáles Moreyra, o, proponer estudios para hacer una psicología del peruano como era la propuesta de Alarcón, o estudiar el desarrollo de los niños sobre todo andinos como fue la propuesta de Pollit. Se tiene que entender que la psicología no es una disciplina vacía, sin contenido ni compromiso social.

\section{Referencias}

Alarcón, R. (1980). Desarrollo y estado actual de la psicología en el Perú. Revista Latinoamericana de Psicología, 12(2), 205-235.

Alarcón, R. (2000). Historia de la psicología en el Perú. De la Colonia a la República. Lima: Editorial Universitaria, Universidad Ricardo Palma.

Alarcón, R. (2006). La contribución de Walter Blumenfeld al desarrollo de la psicología en Perú. Revista de Historia de la Psicología, 27(1), 79-9.

Alarcón, R. (2012). Psicología y testimonio personal. En H. Klappenbach \& R. León (eds.), Historia de la Psicología Latinoamericana en Autobiografías (pp.21-66). Lima, Sociedad Interamericana de Psicología/Editorial Universitaria, Universidad Ricardo Palma.

Andina (2019). BID: Perú debe incrementar inversión en investigación para crecer sostenidamente, Recuperado de https://andina.pe/agencia/noticia-bid-peru-debe-incrementar-inversioninvestigacion-para-crecer-sostenidamente-749912.aspx

Arias, W. (2014). Historia y actualidad en la psicología peruana. En G. Salas. (Ed.), Historias de la Psicología en América del Sur Diálogos y perspectivas (pp.172-204). La Serena, Chile: Nueva Miranda Ediciones

Arias, W. L. (2011). Reseña histórica de la psicología peruana desde la época republicana hasta la actualidad. Revista de Psicología de la UCSP, 1, 73-93.

Arias, W. L. (2012). La contribución de Reynaldo Alarcón a la psicología peruana. Revista de Psicología de la UCSP, 2(2), 71-78.

Banco Mundial (2017). Gasto en investigación y desarrollo (\% del PIB). Recuperado de datos.bancomundial.org/indicador/GB.XPD.RSDV.GD.ZS.

Bermúdez, J. (2013). Investigación científica en el Perú: factor crítico de éxito para el desarrollo del país. Sinergia e Innovación, 1(02), 1-17. Recuperado de http://revistas.upc.edu.pe/index.php/sinergia/article/view/1 30

Caycho, T. (2012). La contribución psicológica de Federico R. León a la psicología peruana y latinoamericana. Revista de Psicología de Arequipa, 2(2), 142-163.

Caycho, T. (2013a). Walter Blumenfeld: Vida y obra de un pionero en el desarrollo de la psicología científica en el Perú. Eureka, 10(2), 216-229.

Caycho, T. (2013b). Aproximación a la obra psicológica de Reynaldo Alarcón y su contribución a la psicología científica en el Perú. Revista de Historia de la Psicología, 34(3), 7-24.

Concytec (2006). Plan Nacional Estratégico de Ciencia, Tecnología e Innovación para la Competitividad y el Desarrollo Humano PNCTI 2006-1021. Lima: Concytec

Jáuregui, D. (2002). La situación de la Psicología en el Perú. Infocop, 14, 31-35.

León, R. (1993a). Walter Blumenfeld a veinticinco años de su muerte. Revista de Psicología, 11(2), $181-194$.

León, R. (1993b). Contribuciones a la historia de la psicología en el Perú. Lima: Concytec.

Medina, S. (26 de noviembre de 2014). Aún cuesta mucho publicar en revistas científicas. Entrevista a Ezequiel Farré. El Comercio. Recuperado de https://assets.elcomercio.pe/noticias/sandro-medina-tovar

Medina, S. (22 de julio de 2015). En el Perú se producen pocos artículos científicos. Entrevista a Josmel Pacheco-Mendoza. El Comercio. Recuperado de https://assets.elcomercio.pe/noticias/sandro-medina-tovar

Merino-Soto, C., \& Salas-Blas, E. (2016). Estructura de las motivaciones y dificultades percibidas para la investigación entre los docentes universitarios: un estudio preliminar. Revista 
Interamericana de Psicologia/Interamerican Journal of Psychology (IJP), 50(2), 161169.

Montero, I., \& León, O. (2005). Sistema de clasificación del método en los informes de investigación en Psicología. International Journal of Clinical and Health Psychology, $5(1), 115-127$.

Rebossio, A. (14 de noviembre de 2013a). ¿Cuánto invierte América Latina en investigación y desarrollo? Diario El País. Recuperado de blogs.elpais.com/.../cuánto-invierte-américa-latina-eninvestigación-y-desarrollo.html.

Rebossio, A. (26 de noviembre de 2013b). Los países latinoamericanos que más y menos invierten. Diario El País. Recuperado de: blogs.elpais.com/eco.../los-países-latinoamericanos-que-más-ymenos-invierten.html.

Rivera, R. (2011). Nuestra hora. Los latinoamericanos en el Siglo XXI. Santiago de Chile: Pearson-Prentice Hall

Salas, E. (2000). Una introducción a la investigación científica. Lima: Tarea Asociación Gráfica Educativa

Taipe, A. (15 de abril de 2016). Entrevista a Gisella Orjeda, Hay ministerios que no nos hacen caso. $\mathrm{El}$ Comercio. Recuperado de https://portal.concytec.gob.pe/index.php/noticias/concytec-en-los-medios/705entrevista-a-gisella-orjeda-hay-ministerios-que-no-nos-hacen-caso-diario-el-comercio15-04-2016

Zanolo, B. (2014). Personas y personajes de la Psicología en el Perú. Dr. Raúl González Moreyra. Avances en Psicología, 22(1), 117-119. 